\section{ORIGINAL} RESEARCH

X. Chen

J. Dai

L. Ai

X. Ru

J. Wang

S. Li

G.S. Young

\title{
Clival Invasion on Multi-Detector CT in 390 Pituitary Macroadenomas: Correlation with Sex, Subtype and Rates of Operative Complication and Recurrence
}

BACKGROUND AND PURPOSE: Clival invasion, a rare but potentially significant complication of pituitary adenoma, is difficult to detect on MR imaging. Because CT is widely used in adjunct guidance of pituitary surgery and it has recently been suggested that preoperative CT may add useful diagnostic information in addition to pituitary MR imaging, we performed the first large cross-sectional imaging study to define the image attributes, clinical correlates, and prognostic implications of clival invasion on CT for pituitary adenoma surgical guidance.

MATERIALS AND METHODS: Preoperative CT images from 390 patients with histopathologically diagnosed pituitary macroadenoma were reviewed retrospectively and classified by the presence and degree of clival invasion. Tumor volume, tumor subtype, patient sex, operative complication, and recurrence rates were compared between groups.

RESULTS: After we corrected for multiple correlations, the most significant independent risk factor for clival invasion was female sex (OR $=3.62, P=.014$, multinomial logistic regression), followed by large tumor volume $(\mathrm{OR}=1.08, P<.001)$, and null-cell subtype $(\mathrm{OR}=5.47, P<.001)$. Larger tumor volume correlated with null-cell subtype (Mann-Whitney $U$ test, $P=.006)$, incidence of clival invasion $(P<.001)$, and extent of clival invasion $(P=.038)$. Clival invasion was associated with a significantly higher ratio of operative complications (15.63\%, $\chi^{2}=7.067, P=.008$ ) and recurrence $\left(57.14 \%, \chi^{2}=10.739, P=.001\right)$.

CoNCLUSIONS: CT detection of clival invasion by pituitary macroadenoma is significantly more common in women, in patients with large tumors, and in patients with null-cell tumors, and it is associated with a higher rate of operative complications and recurrences. Attention to the presence of clival invasion on preoperative $\mathrm{CT}$ and prospective investigation of its prognostic significance are indicated. Attention to this finding on pituitary guidance CT is warranted.

ABBREVIATIONS: $\mathrm{ACTH}=$ adrenocorticotrophic hormone; $\mathrm{Cl}=$ confidence interval; $\mathrm{FSH}=$ folliclestimulating hormone; $\mathrm{GH}=$ growth hormone; $\mathrm{LH}=$ luteinizing hormone; MPR = multiplanar reconstructions; $\mathrm{OR}=$ odds ratio; $\mathrm{PRL}=$ prolactin; $\mathrm{TSH}$ = thyroid-stimulating hormone; $\mathrm{WHO}=$ World Health Organization

A lthough histologically benign, large pituitary adenomas frequently become invasive, infiltrating into surrounding dura, bone, and sinuses. ${ }^{1}$ The most common direction of invasion for pituitary adenoma is suprasellar, followed by infrasellar, lateral, and anterior. ${ }^{2}$ Posterior-inferior invasion into the clivus is rare but presents a technical challenge to surgical resection and may raise the potential risks of cerebrospinal

Received July 23, 2010; accepted after revision September 8.

From the Department of Radiology (X.C., J.D., L.A. S.L.), Beijing Tiantan Hospital, Capital Medical University, Beijing, P. R. China; Departments of Neuroepidemiology (X.R.), and Neuropathology (J.W.), and Neuroimaging Center (L.A.), Beijing Neurosurgical Institute, Bejing, P. R. China; and Department of Radiology (G.S.Y.), Brigham and Women's Hospital and Harvard Medical School, Boston, Massachusetts.

This work was sponsored by the National Natural Science Foundation of China (30770617) and Beijing Sci-Tech Nova Plan B (2007B052). It was also supported by the Ministry of Science and Technology of the People's Republic of China (Project Number: 2007BAl05B08).

Please address correspondence to Lin Ai, MD, Department of Radiology, Beijing Tiantan Hospital, Capital Medical University and Neuroimaging Center of Beijing Neurosurgical Institute, Beijing, P. R. China, 6 Tiantanxili, Dongchen District, Beijing, P R China, 100050; e-mail: ailin136@yahoo.com.cn; or Geoffrey S. Young, MD, Departments of Radiology, Brigham and Women's Hospital and Harvard Medical School, 75 Francis St, Boston MA 02115; e-mail: gsyoung@partners.org

Indicates open access to non-subscribers at www.ajnr.org

DOI 10.3174/ajnr.A2364 rhinorrhea and other complications. The portion of the clivus invaded by pituitary adenomas is most commonly in the anterior/cephalad portion, derived developmentally from the basisphenoid bone. Because of its exquisite soft-tissue contrast for evaluation of sellar tumors, their suprasellar extension, parasellar cavernous sinus invasion, and dural invasion, MR imaging is the primary technique for preoperative assessment of pituitary adenoma and guidance of resection. In many centers, CT is used as an adjunct for guidance because of its superior depiction of bony anatomy, but though it has recently been reported to add useful information to pituitary MR imaging in depicting erosion of the sellar floor and clivus, it is not generally regarded as providing significant diagnostic information. ${ }^{3}$ Our clinical experience with guidance CT suggested that the superior depiction of bony anatomy on CT provides a highly sensitive examination for clival invasion by adenoma, which is often difficult to detect on MR imaging, we designed a retrospective study to assess the performance of CT for operative guidance in 390 patients with pathologically confirmed pituitary macroadenoma. Our analysis was designed to determine the frequency of clival invasion and the association of CT-detected invasion with patients' clinical factors, surgical outcomes, and tumor subtypes. 


\begin{tabular}{|c|c|c|c|c|c|c|c|c|c|}
\hline & Null-Cell & Mixed & FSH & $\mathrm{GH}$ & PRL & ACTH & $\mathrm{TSH}$ & LH & Total \\
\hline \multicolumn{10}{|l|}{ Included } \\
\hline Female & 59 & 30 & 25 & 19 & 18 & 12 & 12 & 7 & $182(47 \%)$ \\
\hline Male & 83 & 38 & 16 & 21 & 15 & 21 & 9 & 5 & $208(53 \%)$ \\
\hline Total & 142 (36\%) & $68(17 \%)$ & $41(11 \%)$ & $40(10 \%)$ & 33 (8\%) & $33(8 \%)$ & $21(5 \%)$ & $12(3 \%)$ & \\
\hline \multicolumn{10}{|l|}{ Excluded } \\
\hline Female & 19 & 10 & 6 & 7 & 7 & 4 & 3 & 1 & $57(53 \%)$ \\
\hline Male & 20 & 9 & 8 & 5 & 4 & 3 & 1 & 1 & $51(47 \%)$ \\
\hline Total & 39 (36\%) & $19(18 \%)$ & $14(13 \%)$ & $12(11 \%)$ & $11(10 \%)$ & $7(6 \%)$ & $4(4 \%)$ & $2(2 \%)$ & \\
\hline
\end{tabular}

\section{Materials and Methods}

\section{Patient Demographics and Exclusion Criteria}

The local institutional ethics committee approved this study and granted a waiver of informed consent. CT and clinical data were retrieved retrospectively for 498 consecutive patients who underwent pituitary surgery at a leading Chinese neurosurgical hospital between September 2008 and April 2009 and whose tumors fulfilled the WHO histopathologic 2007 criteria for the diagnosis of pituitary adenoma. ${ }^{4}$ One hundred eight patients (22\%) were excluded on the basis of the following criteria: 1) absence of preoperative CT scanning (55 patients), 2) preoperative CT scans lacking MPR (24 patients), 3) pituitary microadenoma (15 patients), 4) recurrent pituitary adenoma following prior resection at other institutions (8 patients), and 5) preoperative radiation therapy ( 6 patients). Three hundred ninety patients ( 208 men, 182 women; mean age, $45.78 \pm 12.53$ years, range, $18-75$ years) were included in further analysis.

\section{Image Acquisition}

All patients included in this study had undergone preoperative CT of the sella turcica with a standard institutional protocol for assessment of bone invasion within 2 weeks before surgery. Patients were scanned on a 16-section CT scanner (Somatom Sensation; Siemens, Erlangen, Germany) in the supine position. The scanner gantry was angled parallel to a line connecting the outer canthus of the eye to the external auditory meatus (canthomeatal line), and imaging was performed from the superior margin of the odontoid process to the level of foramen of Monro. The protocol consisted of axial scanning with production of sagittal and coronal MPR. Acquisition parameters were the following: $120 \mathrm{KV}$; $310 \mathrm{~mA}$; and $0.8-\mathrm{mm}$ collimation. Axial reconstructions with an FOV of $250 \mathrm{~mm}$, matrix of $512 \times 512$, section thickness and gap of $0.75 \mathrm{~mm}$ were produced and used to produce sagittal and coronal MPR. Section thickness varied slightly according to the size of the adenoma, with a thickness of 2-3 $\mathrm{mm}$ and gap of 2-4 $\mathrm{mm}$.

\section{Image Analysis}

The preoperative CT images were retrospectively and independently analyzed by 2 neuroradiologists (L.A., 13 years of experience, and S.L., 15 years of experience) who were blinded to the patients' histories, physical examination findings, results of serum tests, tumor subtypes, and detection of gross invasion at surgery. Manual measurement of maximal tumor diameters (millimeters) was performed in cephalocaudal, left-right, and anteroposterior directions, by using the scanner console software. For each tumor, the measurement was performed twice and the average value was recorded as the final diameter. The tumor volume (cubic centimeters) was calculated according to the following formula: $\mathrm{V}_{\text {lesion }}=(\pi / 6) \times$ height $\times$ width $\times$ length. ${ }^{5}$ The volume was classified by median and quartile (25\%-75\%) values. Invasion of the clivus was diagnosed when there was a focal or widespread defect in the cortex of the clivus and decreased attenuation in the underlying trabecular bone. When the cortex was continuous and no decrease in underlying trabecular bone attenuation was seen, the process was regarded as bony remodeling and not classified as clival invasion. Clival invasion was classified as focal when the width of the low-attenuation area was less than half of the width of the clivus on the same section or less than one-third of the height of the clivus on sagittal MPR. All others cases of invasion were classified as extensive.

\section{Clinical and Histopathologic Diagnosis}

Results of endocrine panel testing performed 1 week before surgery were retrieved. This panel included triiodothyronine, tetraiodothyronine, supersensitive TSH, dissociated triiodothyronine and tetraiodothyronine, PRL, LH, FSH, estradiol, progesterone, GH, ACTH, cortisol hydrocortisone, and testosterone. Routine diagnostic histopathologic analysis including interpretation of standard hematoxylin-eosin-stained sections was retrieved and used to confirm the diagnosis of pituitary adenoma. Immunohistochemical staining results were retrieved to subclassify the tumors by the presence of secretory granules or precursors for FSH, GH, PRL, ACTH, TSH, or LH. Tumors demonstrating no staining for any of the above hormones or precursors were classified as null-cell adenomas. Otherwise, they were regarded as secreting adenomas.

\section{Statistical Analysis}

Statistical analysis was performed on commercial statistical software (Statistical Package for the Social Sciences, Version 10.0; SPSS, Chicago, Illinois). Binary and multinomial logistic regression was used to test the influence of patient age, sex, tumor subtype, and tumor volume on the rates of clival invasion. The $\chi^{2}$ test was used to analyze the association of patient sex, pathologic subtype, operative approach and complications, and recurrence with clival invasion. This test was also used to analyze the difference in frequency of null-cell tumors between male and female patients. The 2 independent-samples $t$ test was used to test the difference in follow-up periods between the patients with clival destruction and those without. Because the tumor volume data did not include a normal distribution, the Mann-Whitney $U$ test was used to compare tumor volumes between male and female patients, between null-cell and secreting macroadenomas, between tumors with and without clival invasion, and between tumors with focal and extensive clival invasion. The correlation between tumor volume and pathologic subtypes (null-cell versus secreting tumors) was analyzed by Spearman correlative analysis. A $P$ value $<.05$ was considered statistically significant.

\section{Results}

Table 1 lists that pathologic subtypes of tumors in the 390 patients included in the study and the 108 excluded patients. 


\begin{tabular}{|c|c|c|c|c|c|c|c|c|c|c|}
\hline \multirow[b]{2}{*}{$\begin{array}{l}\text { Clival } \\
\text { Invasion }\end{array}$} & \multicolumn{3}{|c|}{ Sex } & \multicolumn{2}{|c|}{ Age } & \multicolumn{3}{|c|}{ Pathologic Subtype } & \multicolumn{2}{|c|}{ Volume } \\
\hline & Male & Female & $\begin{array}{c}\chi^{2}, \\
P \text { Value }\end{array}$ & Mean (yr) & $\begin{array}{c}2 \text { Independent- } \\
\text { Samples } \\
t \text { Test }\end{array}$ & Null-Cell & Secreting & $\begin{array}{c}\chi^{2} \\
P \text { Value }\end{array}$ & $\left(\right.$ mean, $\left.\mathrm{cm}^{3}\right)$ & $\begin{array}{c}\text { Mann-Whitney } \\
\text { Test, } \\
P \text { Value }\end{array}$ \\
\hline$\overline{\text { With }}$ & $7(1.79 \%)$ & $25(6.41 \%)$ & 13.861, & $49.84 \pm 9.25$ & $P=.972$ & $23(5.90 \%)$ & $9(2.31 \%)$ & 18.937 & $16.45 \pm 22.18$ & Significant, \\
\hline Without & $201(51.54 \%)$ & $157(40.26 \%)$ & $P<.001$ & $45.61 \pm 12.39$ & & $119(30.51 \%)$ & $239(61.28 \%)$ & $P<.001$ & $4.94 \pm 6.18$ & $P<.001$ \\
\hline Summary & $208(53.33 \%)$ & $182(46.67 \%)$ & & $45.78 \pm 12.53$ & & $142(36.41 \%)$ & $248(63.59 \%)$ & & $5.55 \pm 7.43$ & \\
\hline
\end{tabular}

\begin{tabular}{|c|c|c|c|c|c|c|}
\hline & \multicolumn{3}{|c|}{ Binary Logistic Regression } & \multicolumn{3}{|c|}{ Multinomial Logistic Regression } \\
\hline & B-Value & OR $(95 \% \mathrm{Cl})$ & $P$ Value & B-Value & OR $(95 \% \mathrm{Cl})$ & $P$ Value \\
\hline Age & 0.029 & 1.030 (0.999-1.062) & .061 & 0.015 & $1.015(0.979-1.053)$ & .419 \\
\hline Female sex & 1.349 & $3.852(1.548-9.585)$ & .004 & 1.286 & 3.620 (1.298-10.098) & .014 \\
\hline Null-cell subtype & 1.537 & $4.648(2.088-10.350)$ & $<.001$ & 1.067 & $2.907(1.142-7.400)$ & .025 \\
\hline Large volume & 0.096 & $1.101(1.065-1.138)$ & $<.001$ & 0.078 & $1.081(1.047-1.117)$ & $<.001$ \\
\hline
\end{tabular}

\begin{tabular}{|c|c|c|c|c|c|c|c|c|}
\hline & \multicolumn{2}{|c|}{ Operation Approaches } & \multicolumn{2}{|c|}{ Resection Extent } & \multirow{2}{*}{$\begin{array}{l}\text { Operative } \\
\text { Complications }\end{array}$} & \multicolumn{2}{|c|}{ Follow-Up } & \multirow[b]{2}{*}{ Recurrence } \\
\hline & Transsphenoidal & Transfrontal & Gross Total & Subtotal & & Cases & Period $(\mathrm{mo})$ & \\
\hline Clival destruction & $26 / 32(81.25 \%)$ & $6 / 32(18.75 \%)$ & 0 & 32 & $5 / 32(15.63 \%)$ & 14 & $6.78 \pm 2.91$ & $8 / 14(57.14 \%)$ \\
\hline Without clival destruction & $330 / 358(92.18 \%)$ & $28 / 358(7.82 \%)$ & 99 & 259 & $13 / 358(3.63 \%)$ & 56 & $8.58 \pm 3.16$ & $7 / 56(12.50 \%)$ \\
\hline $\begin{array}{l}\chi^{2} \\
P\end{array}$ & \multicolumn{2}{|c|}{$\begin{aligned} \chi^{2} & =3.142 \\
P & =.076\end{aligned}$} & & & $\begin{array}{c}\chi^{2}=7.067 \\
P=0.008\end{array}$ & & $P<.001^{\mathrm{a}}$ & $\begin{array}{c}\chi^{2}=10.739 \\
P=.001\end{array}$ \\
\hline
\end{tabular}

a Two independent-samples $t$ test.

The percentage of women was not significantly different between the included (47\%) and excluded (48\%) groups.

The included and excluded populations were not different $\left(\chi^{2}=1.265, P=.261\right)$.

Thirty-two of 390 patients (8.21\%) had invasion of the clivus at surgery (Table 2). All macroadenomas with clival invasion underwent subtotal resection: $26(81 \%)$ by a transsphenoidal approach and $6(19 \%)$ by a transfrontal approach. Of the remaining 358 tumors without clival invasion, 330 (92\%) underwent transsphenoidal surgery and 28 (8\%), transfrontal surgery. This resulted in $99(28 \%)$ gross total resections and 259 (72\%) subtotal resections. Operative complications for the 390 tumors included infarction of the frontal lobe, temporal lobe, and genu of the corpus callosum; intraventricular hemorrhage; subarachnoid hemorrhage; frontal lobe parenchymal hematoma; and cerebrospinal rhinorrhea.

One hundred forty-two of 390 cases (36\%) were pathologically classified as null-cell adenomas. Twenty of the 142 nullcell adenomas $(14 \%)$ showed elevated hormone levels: supersensitive TSH $(n=1)$, serum PRL $(n=17)$, both serum PRL and cortisol $(n=1)$, and both serum PRL and supersensitive TSH $(n=1)$. Of the 17 patients with null-cell adenomas with elevated serum PRL, 1 female patient showed diffuse clival invasion. Because the conflict between the pathologic subtype and secretory status in these patients rendered their classification equivocal, a subanalysis was performed excluding them. Analysis of the remaining 122 unequivocal null-cell macroadenomas still demonstrated larger size $(P=.010)$ and more frequent clival invasion $\left(\chi^{2}=22.101, P<.001\right)$ than the 248 secreting adenomas and a female predominance of clival inva$\operatorname{sion}\left(\chi^{2}=13.495, P<.001\right)$.

Logistic regression analysis) revealed a significant correlation between clival invasion and female sex $\left(\chi^{2}=13.861\right.$,
$P<0.001)$, large tumor size $(P<0.001)$ and null-cell pathologic subtype $\chi^{2}=18.937, P<0.001$ (Tables 2 and 3 ). There was no significant difference between the size of tumors in women $\left(5.73 \pm 7.22 \mathrm{~cm}^{3}\right)$ and men $\left(5.92 \pm 7.63 \mathrm{~cm}^{3}, P=\right.$ .174 ) or between the incidence of pituitary macroadenoma in women and men (Table 2). The correlation between clival invasion and null-cell subtype was slightly strengthened after exclusion of the 20 false null-cell tumors $(\mathrm{OR}=5.47$; range, 2.45-12.21; $P<.001$, binary logistic regression).

Although null-cell macroadenomas were less common in this series than secreting tumors, statistical analysis demonstrated a higher rate of clival invasion in null-cell macroadenomas $\left(\chi^{2}=18.937, P<.001\right.$; Table 2$)$. Null-cell macroadenomas were found in $32 \%(59 / 182)$ of the female patients and $40 \%(83 / 208)$ of the male patients. The difference was not significant $\left(\chi^{2}=2.350, P=.125\right)$.

Although the operative approach was similar between patients whose tumors had clival invasion on CT and those whose did not, gross total resection was less frequent and operative complications were significantly more common in patients with clival invasion on CT $(16 \%, 5 / 32)$ (Table 4). Despite a similar period of follow-up, recurrence was seen in a higher ratio of patients with clival invasion $(57 \%, 8 / 14)$.

Larger tumor volume correlated with higher frequency of clival invasion (Table 3). The 32 tumors with clival invasion were significantly larger than those in the 358 patients without invasion (Table $2, P<.001$ ), and 25 tumors with extensive clival invasion $\left(18.90 \pm 9.05 \mathrm{~cm}^{3}\right.$, Fig $\left.1 A,-B\right)$ were significantly larger than those in the 7 patients with localized invasion $\left(14.04 \pm 3.35 \mathrm{~cm}^{3}, P=.038\right.$, Fig. $\left.2 A,-B\right)$. In addition, the mean volume of the 142 null-cell macroadenomas (7.69 \pm $11.37 \mathrm{~cm}^{3}$ ) was significantly larger than that of the 248 secreting tumors $\left(4.87 \pm 6.59 \mathrm{~cm}^{3}, P=.006\right)$. Nevertheless, despite 

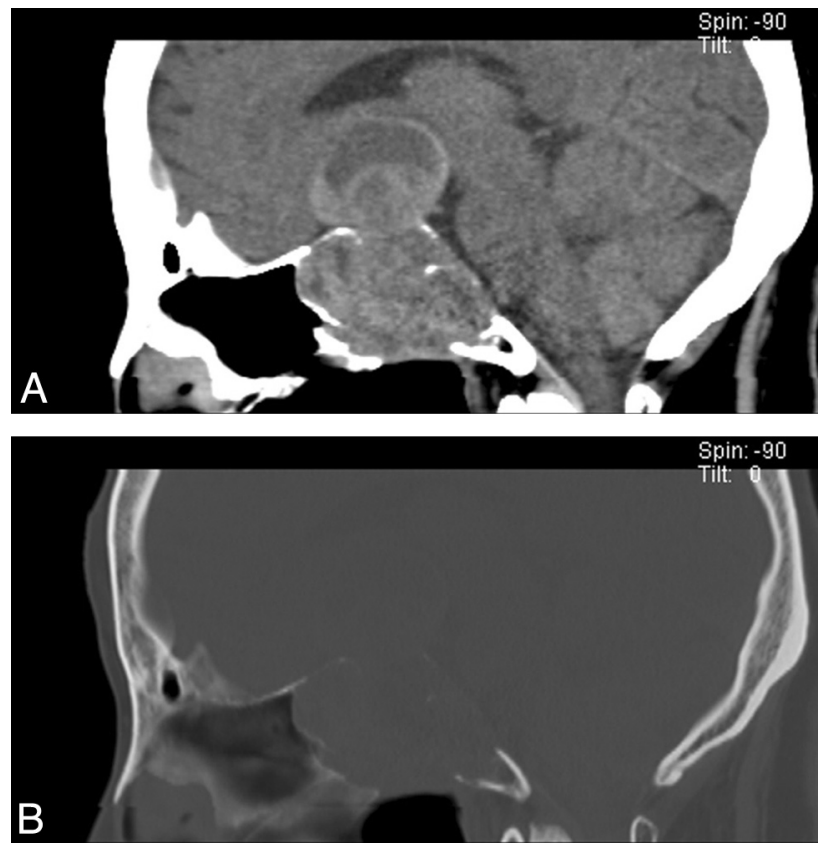

Fig 1. Soft-tissue $(A)$ and bone algorithm $(B)$ sagittal MPR in a 55-year-old female patient with null-cell pituitary macroadenoma, producing extensive clival invasion. The tumor volume is $35.40 \mathrm{~cm}^{3}$.
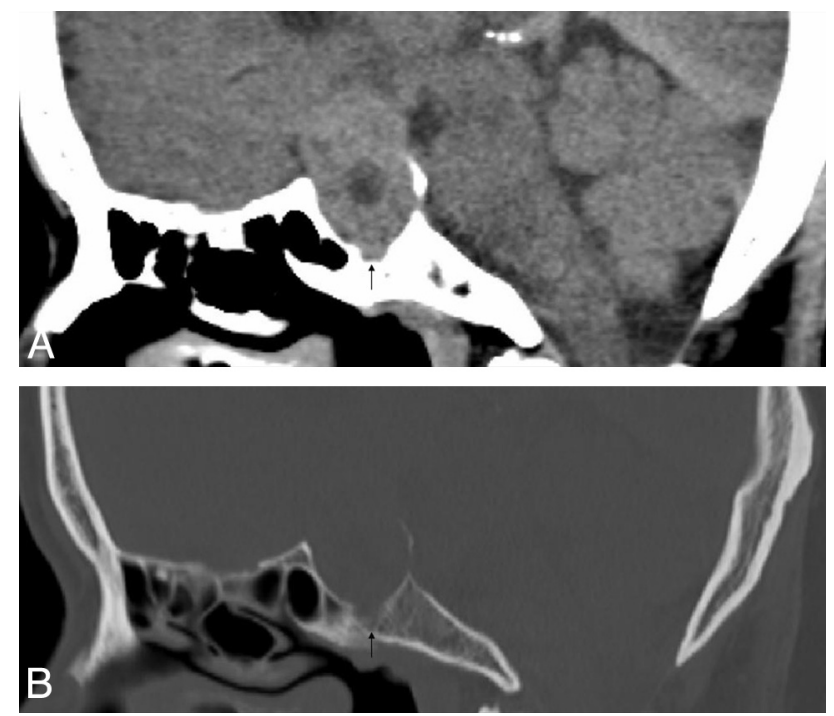

Fig 2. Soft-tissue $(A)$ and bone algorithm $(B)$ sagittal MPR in a 43-year-old male patient with a null-cell pituitary macroadenoma, producing focal clival invasion (black arrow). The tumor volume is $17.02 \mathrm{~cm}^{3}$.

correction for the significant correlation of tumor volume with pathologic subtype $(r=-0.247, P<.001)$, an independent correlation persisted between tumor volume and clival invasion $(b=-.050, P<.001, \mathrm{OR}=0.951)$.

\section{Discussion}

The invasive nature of pituitary macroadenoma is well documented, but while invasion of the cavernous sinuses and carotid arteries and along the dura is common, invasion of the clivus is relatively rare. The few reports published to date have not reported series of sufficient size to establish the characteristic clinical, pathologic, and imaging attributes of clival invasion by pituitary adenoma. ${ }^{6-9}$
In our study population, most patients with macroadenoma presented in the fourth and fifth decades of life; the frequency and size of macroadenoma were nearly equal in men $\left(53.33 \%\right.$ of cases; $\left.5.92 \pm 7.63 \mathrm{~cm}^{3}\right)$ and women $(46.67 \%$; $\left.5.73 \pm 7.22 \mathrm{~cm}^{3}\right)$; the proportion of null-cell pituitary macroadenoma was $36.4 \%(142 / 390)$. These population statistics are similar to previous reports, ${ }^{10-13}$ suggesting that this sample, though retrospective, may be a relatively good representation of the general population of patients with adenoma.

Among patients with macroadenoma in this study, female sex was the strongest risk factor for clival invasion $(\mathrm{OR}=3.62$, $P=.014)$. The multinomial logistic regression also demonstrated a female predominance $(P<.001)$. This finding came as a surprise because the incidence of macroadenoma and tumor size was similar between women and men in our series and because it is discordant with a previous study suggesting that macroadenomas and invasive adenomas are significantly more frequent in men. ${ }^{14}$ The discrepancy with this previous report seems likely to be due to the type of invasion studied. In addition to bone invasion, Qian et $\mathrm{al}^{14}$ included invasion of the sphenoid sinuses, cavernous sinuses, and brain in their study, whereas we focused exclusively on invasion of the clivus.

Larger tumor volume was the second most important risk factor after female sex $(\mathrm{OR}=1.08, P<.001)$. When analyzed as a group, the 32 tumors with clival invasion were larger than the 358 tumors without clival invasion $(P<.001)$, and the 25 tumors with extensive invasion were larger than those in the 7 tumors with localized invasion $(P=.038)$. This relationship parallels reports of increased cavernous sinus invasion in larger tumors. ${ }^{15-17}$

The finding that clival invasion is more frequent in null-cell adenomas (OR, 4.65) even after correction for larger average tumor volume $(P<.001 ; \mathrm{OR}, 0.951)$ is intriguing. It suggests that the null-cell phenotype is an independent risk factor for clival invasion, raising the possibility that the null-cell phenotype represents a marker for less differentiated and thus more aggressive tumors. Given the significance of the null-cell phenotype as a risk factor, it is important to consider the implications of the 20 patients in this group with apparent conflict between laboratory analysis and pathology. While we cannot be certain, one likely explanation would seem to be that the tumors were heterogeneous and that the failure to detect secretory staining was a result of histologic sampling error, which would imply that these patients had a mixed phenotype. The persistence of this correlation after exclusion of these 20 equivocal patients supports the notion that null cell phenotype is an independent risk factor for clival invasion.

Female sex, the strongest of the 3 identified risk factors for clival invasion, was also independent of both large tumor size and null-cell subtype. This is supported by the observation that male and female patients in our series did not show a significant difference in macroadenoma incidence, tumor volume $(P=.174)$, or percentage of null-cell tumors $(P=.125)$. This finding could imply either that female patients are prone to more aggressive tumors or that some undefined feature of female patient physiology predisposes to clival invasion. Either effect-tumor- or host-related-seems likely to be due to either genetic or hormonal factors and may deserve further investigation with genetics and in vitro tumor cell culture.

Although our study was not designed to investigate the 
surgical implications of invasion, the findings of lower rates of gross total resection and higher rates of operative complications and recurrence underscore the clinical significance of clival invasion in pituitary macroadenoma. This implies that further study of therapeutic technique may be warranted in this patient group. Although invasion of pituitary tumors into the clivus has been described, ${ }^{9-18}$ mostly in the form of case reports, ${ }^{7,8,19}$ to our knowledge, this is the first study designed to systematically define the clinical and histologic correlates of clival invasion on CT in a large patient cohort.

The major weaknesses of our study include its retrospective design and the possibility of selection bias inherent to clinical case series. Although the possibility of inadvertent selection bias is impossible to completely exclude in a retrospective series, the overall sex and subtype mix of the included population is consistent with previously published series and is wellbalanced by sex and endocrine status. Similarly, given the relatively large number of patients who had to be excluded from analysis because of inadequate imaging, neither the percentage of women $(48 \%)$ nor the percentage of null-cell adenomas (36\%) was significantly different in the excluded group compared with group of included patients analyzed.

An additional weakness in the study that must be noted is that the $8 \%(32 / 390)$ rate of clival invasion we found on CT may underestimate the true rate because we missed microscopic invasion, the clinical significance of which is unknown. In addition, the tumor volumes we report, though comparable across the patients in the study, may overestimate the absolute tumor volume because the formula used to calculate tumor volume from the 3 measured maximum diameters assumes a roughly spheric lesion, but in fact, the shape of many of the lesions was very irregular. This could result in an underestimate of the strength of the correlation between tumor size and invasion. Finally, the relatively short follow-up period and relatively small fraction of included patients who were available for follow-up somewhat limit the reliability of conclusions about recurrence rates.

Furthermore, the lack of comparison MR imaging precludes us from concluding definitively that the observed bony erosion of the clivus itself is responsible for the observed association with higher complication and recurrence rates. Because it is likely that the larger tumors associated with clival invasion may also be associated with higher rates of mass effect on and invasion of the cavernous sinus, brain, or vessels, it is possible that the observed associations with higher rates of surgical complications and residual/recurrent disease are mediated by these other tumor attributes rather than by clival invasion per se. A retrospective follow-up analysis in patients who also had MR imaging is planned to address this possibility. This planned analysis may also help to address an additional limitation of the study. Although in general, CT evaluation of bony invasion has been shown to add to the information provided by MR imaging, the extent to which MR imaging might have provided information of similar diagnostic importance in our cohort remains to be determined.

Even if, as we expect, the additional bony detail obtained by CT adds to the overall preoperative diagnostic impression, it remains to be seen whether this additional information will prove clinically significant enough to justify additional scanning in patients not already getting CT for surgical guidance. To demonstrate this, a prospective trial of CT as an adjunct to MR imaging for surgical guidance would be required and may be justifiable.

\section{Conclusions}

Clival invasion in pituitary macroadenoma was detected by preoperative CT in $8.21 \%$ of patients. Multivariate analysis revealed female sex to be the most important risk factor for clival invasion, followed by larger tumor volume and null-cell subtype. As expected, larger tumor size correlated with more extensive invasion. It remains to be seen whether the effect of female sex is mediated by tumor or patient genetic or hormonal factors and what the underlying molecular basis of increased invasion in null-cell adenomas may be. Although the primary goal of our study was not to assess outcome, CT evidence of clival invasion was associated with a lower rate of complete resection, a higher rate of operative complications, and a higher rate of recurrence in our series. Further retrospective analysis is indicated to determine if this association is independent of findings of soft-tissue invasion detectable on MR imaging, and a prospective study may be indicated to confirm whether these associations are found across centers, whether the additional information provided by CT alters management or outcome, and whether alternative therapeutic approaches should be considered in patients with macroadenoma with clival invasion on CT.

\section{References}

1. Asa SL. Practical pituitary pathology: what does the pathologist need to know? Arch Pathol Lab Med 2008;132:1231-40

2. Luo CB, Teng MM, Chen SS, et al. Imaging of invasiveness of pituitary adenomas. Kaohsiung J Med Sci 2000;16:26-31

3. Miki Y, Kanagaki M, Takahashi JA, et al. Evaluation of pituitary macroadenomas with multidetector-row CT (MDCT): comparison with MR imaging. Neuroradiology 2007;49:327-33

4. Louis DN, Ohgaki H, Wiestler OD, et al. The 2007 WHO classification of tumours of the central nervous system. Acta Neuropathol 2007;114:97-109. Epub 2007 Jul 6. Erratum in: Acta Neuropathol 2007;114:547

5. Stadlbauer A, Buchfelder M, Nimsky C, et al. Proton magnetic resonance spectroscopy in pituitary macroadenomas: preliminary results. J Neurosurg 2008;109:306-12

6. Al-Mefty O, Kadri PA, Hasan DM, et al. Anterior clivectomy: surgical technique and clinical applications. J Neurosurg 2008;109:783-93

7. Goel A, Phalke U, Cacciola F, et al. Giant pituitary adenoma invading the clivus. Neurol India 2005;53:105-07

8. Coulson CJ, Siddiq MA, Johnson AP. Empty sella syndrome associated with a hyperfunctioning microadenoma invading the clivus. $\mathrm{Br} J$ Neurosurg 2007;21:623-25

9. Wong K, Raisanen J, Taylor SL, et al. Pituitary adenoma as an unsuspected clival tumor. Am J Surg Pathol 1995;19:900-03

10. Osamura RY, Kajiya H, Takei M, et al. Pathology of the human pituitary adenomas. Histochem Cell Biol 2008;130:495-507

11. Goh KP, Lee HY, Rajasoorya RC. Triple jeopardy in the pituitary. Pituitary 2008;11:331-36

12. Ferreira JE, de Mello PA, de Magalhães AV, et al. Non-functioning pituitary adenomas: clinical features and immunohistochemistry [in Portuguese]. Arq Neuropsiquiatr 2005;63:1070-78. Epub 2005 Dec 15

13. Buurman $H$, Saeger W. Subclinical adenomas in postmortem pituitaries: classification and correlations to clinical data. Eur J Endocrinol 2006;154:753-58

14. Qian ZR, Sano T, Asa SL, et al. Cytoplasmic expression of fibroblast growth factor receptor-4 in human pituitary adenomas: relation to tumor type, size, proliferation, and invasiveness. J Clin Endocrinol Metab 2004;89:1904-11

15. Hagiwara A, Inoue Y, Wakasa K, et al. Comparison of growth hormone-producing and non-growth hormone-producing pituitary adenomas: imaging characteristics and pathologic correlation. Radiology 2003;228:533-38

16. Selman WR, Laws ER Jr, Scheithauer BW, et al. The occurrence of dural invasion in pituitary adenomas. J Neurosurg 1986;64:402-07

17. Scheithauer BW, Kovacs KT, Laws ER Jr, et al. Pathology of invasive pituitary tumors with special reference to functional classification. I Neurosurg 1986;65:733-44

18. Zhao B, Wei YK, Li GL, et al. Extended transsphenoidal approach for pituitary adenomas invading the anterior cranial base, cavernous sinus, and clivus: a single-center experience with 126 consecutive cases. J Neurosurg 2010;112:108-17

19. Anand VK, Osborne CM, Harkey HL 3rd. Infiltrative clival pituitary adenoma of ectopic origin. Otolaryngol Head Neck Surg 1993;108:178-83 